\title{
The New FNAL Muon g-2 Experiment
}

\author{
Mark Lancaster* (on behalf of the FNAL E989 collaboration) \\ Department of Physics and Astronomy, UCL, London, WCIE 6BT, UK. \\ E-mail: m.lancaster@ucl.ac.uk
}

\begin{abstract}
The most recent measurement of the anomalous magnetic moment of the muon by the BNL E821 experiment differs from the Standard Model prediction by approximately 3.5 standard deviations. This may be indicative of new physics beyond the Standard Model or a statistical fluctuation. In this talk a new experiment, FNAL E989, is described that is seeking to improve on the precision of the BNL measurement by more than a factor of 4 and so resolve this issue. This precision is sufficient to establish evidence for new physics beyond the Standard Model at more than $5 \sigma$ should the present anomaly be confirmed.
\end{abstract}

International Conference on the Structure and the Interactions of the Photon including the 20th International Workshop on Photon-Photon Collisions and the International Workshop on High Energy Photon Linear Colliders

20 - 24 May 2013

Paris, France.

\footnotetext{
*Speaker.
} 


\section{Introduction}

The magnetic moment of the muon is generally defined by the quantity $g-2$ or $a_{\mu}=\frac{1}{2}(g-2)$ where $a_{\mu}$ is referred to as the anomalous contribution i.e. the part arising from higher order interactions not defined in the Dirac equation, where $g$ is exactly 2. In the Standard Model (SM) $a_{\mu}$ receives higher order contributions from QED, electroweak (EWK) and hadronic processes. Experimentally $a_{\mu}$ is the most precisely measured quantity at an accelerator. The BNL E821 experiment determined $a_{\mu}$ to a precision of $0.54 \mathrm{ppm}$ [1] which is somewhat larger than the precision of the SM prediction of $0.42 \mathrm{ppm}$ [2]. The measured and SM value differ by approximately 3.5 standard deviations and this has resulted in over 2,600 publications citing the BNL result, with explanations of the discrepancy ranging from the prosaic to the esoteric. The motivation for a new measurement is to investigate this discrepancy with a greater precision. The new FNAL g-2 experiment will measure $a_{\mu}$ to a precision of $0.14 \mathrm{ppm}$ and the uncertainty on the SM prediction is expected to reduce by almost $50 \%$ on the same timescale. Even without an improvement in the SM estimate, the improved precision expected from the new FNAL experiment is sufficient to establish a discrepancy with a significance beyond 5 standard deviations should the present difference be maintained and beyond eight standard deviations if the improvement in the SM estimate is also realised.

The $a_{\mu}$ measurement complements the measurements being undertaken at the LHC. If new physics is observed at the LHC then the $a_{\mu}$ measurement will be essential in establishing the integrity of any models invoked to explain new phenomena, and moreover can be used to determine model parameters and resolve degeneracy in models that the LHC data alone cannot. For example some SUSY parameters, such as those describing slepton and Higgs mixing, cannot be determined from LHC data alone, and marked differences in the muon magnetic moment are predicted between different extra dimension models. Moreover the measurement also has sensitivity to chirality-flipping interactions and low energy phenomena which the LHC has limited sensitivity to. If no new physics is observed at the LHC and the magnetic moment is found to be consistent with the SM then this again severely constrains the class of models that can still be considered as candidates to explain the deficiencies of the SM.

\section{Standard Model Value for $a_{\mu}$}

By far the most dominant contribution to the SM value of $a_{\mu}$ is the simplest higher order QED contribution, first calculated by Schwinger in 1948 [3], which contributes exactly $\frac{\alpha}{2 \pi}$ to $a_{\mu}$. The QED contribution has recently been determined, over a 9-year period, to 5-loops [4] from a calculation of 12,672 Feynman diagrams and independently cross-checked to 4-loops [5]. The uncertainty on the QED prediction, predominantly from the uncertainty in $\alpha$, is $0.3 \mathrm{ppb}$ which is far below the $0.14 \mathrm{ppm}$ precision anticipated from the FNAL E989 experiment and some 6,000 times smaller than the present discrepancy with respect to the SM. The EWK contribution [6], recently extended to a complete 2-loop calculation and incorporating the measured value for the Higgs boson mass, is negligible beyond NLO and its uncertainty, $0.01 \mathrm{ppm}$, is also significantly below the expected measurement accuracy. The EWK contribution from diagrams involving the Higgs is also far below the measurement accuracy. However the total EWK contribution of $1.3 \mathrm{ppm}$ is larger than the present and future measurement accuracy and as such the $a_{\mu}$ measurement is an 
incisive probe of the SM's EWK sector. The hadronic contribution has an uncertainty (0.42 ppm) comparable with the present measured uncertainty and three times the anticipated precision of the FNAL E989 experiment. A significant theoretical effort is already underway to reduce this uncertainty through improved lattice calculations and the incorporation of new low-energy $e^{+} e^{-}$ measurements. These are discussed in detail in several contributions to these proceedings [7-12]. It is expected on the timescale of the FNAL $a_{\mu}$ measurement that the uncertainty on the hadronic uncertainty will have halved allowing the more precise measurement to better probe for evidence of new physics. The different SM contributions to $a_{\mu}$ are shown in Fig. 1 along with the projected uncertainty of the FNAL measurement and the size of the current discrepancy between the BNL E821 measurement and the SM prediction. The discrepancy is almost twice the magnitude of the EWK contribution to $a_{\mu}$.

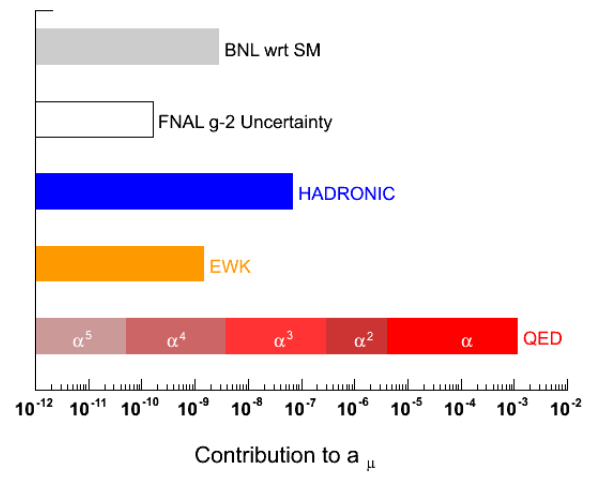

Figure 1: The size of the SM contributions to $a_{\mu}$ along with the projected uncertainty on the FNAL measurement and the size of the current discrepancy between the BNL E821 measurement and the SM prediction.

It is worth noting that the recent measurement of the Higgs boson mass also affords a crosscheck of the SM estimate of the largest hadronic contribution to $a_{\mu}$ : the leading order hadronic vacuum polarisation contribution. This contribution is determined from low energy $e^{+} e^{-}$cross section data via a dispersion integral. The same cross section data also appears in a dispersion integral that determine the hadronic corrections to the fine coupling constant and the value of the fine coupling constant in turn has an influence on the Higgs mass returned in the fits to EWK data. The present fits to EWK data return a Higgs mass of $94_{-22}^{+25} \mathrm{GeV}$ [13] in good agreement with the value measured by ATLAS and CMS, highlighting that the low energy $e^{+} e^{-}$data is reliable. Indeed if the measured Higgs boson mass is used to constrain the hadronic contribution to the fine coupling constant then the predicted leading order hadronic vacuum polarisation contribution to $a_{\mu}$ reduces [14] and the discrepancy between the BNL measurement and the SM prediction of $a_{\mu}$ is increased.

\section{Models of new physics probed by g-2}

One of the principle motivations for this measurement is to probe for evidence of new physics 
beyond the SM (BSM) and so constrain BSM models or help resolve ambiguity in the model parameters should new physics be observed in the analysis of the higher energy data of the LHC: the analysis of which will coincide with the first data from the new FNAL experiment. The contribution of new physics to $a_{\mu}$ come from CP and flavour-conserving interactions which are sensitive to and potentially enhanced by chirality flips. Many high-energy collider observables are insensitive to chirality flips and many other low-energy observables are chirality-flipping but flavor-violating (bor K-decays, $\mu \rightarrow$ e conversion, etc.) or CP-violating (electric dipole moments) and so the $a_{\mu}$ measurement represents a unique and so complementary probe of new physics.

The role of $a_{\mu}$ as a discriminator between very different SM extensions can be characterised by a relation discussed by Czarnecki and Marciano [15] that holds in a wide range of models as a result of the chirality-flipping nature of $a_{\mu}$. BSM physics associated with a mass scale of $\Lambda$ will modify the muon mass by $\delta m_{\mu}^{\mathrm{BSM}}$ and $a_{\mu}$ by $\delta a_{\mu}^{\mathrm{BSM}}$ and these two effects are related via:

$$
\delta a_{\mu}^{\mathrm{BSM}}=O(1) \times\left(\frac{m_{\mu}}{\Lambda}\right)^{2} \times\left(\frac{\delta m_{\mu}^{\mathrm{BSM}}}{m_{\mu}}\right) .
$$

The ratio $\mathrm{C}^{\mathrm{BSM}} \equiv \frac{\delta m_{\mu}^{\mathrm{BSM}}}{m_{\mu}}$ is typically between $O\left(\frac{\alpha}{4 \pi}\right)$ (for perturbative contributions to the muon mass) and $O(1)$ (if the muon mass is essentially due to radiative corrections). The contributions to $a_{\mu}$ are highly model dependent: hence the motivation for a precise measurement. The same equation applies for the electron $a_{e}$ and so while $a_{e}$ is measured to far higher precision $(0.3 \mathrm{ppb})$ than will ever be possible for the muon, its sensitivity to new physics is limited, due to the $m_{\ell}$ weighting, to scales below $100 \mathrm{MeV}$ while muon measurements can probe new physics from scales of $20 \mathrm{MeV}$ up to and beyond the electroweak scale.

In the models predicting large values of $\mathrm{C}^{\mathrm{BSM}}(\sim 1)$ that include extended technicolour, the muon mass is generated by radiative effects at some scale $\Lambda$ and the BSM contribution to $a_{\mu}$ can be very large i.e. $1100 \times 10^{-11}\left(\frac{1 \mathrm{TeV}}{\Lambda}\right)^{2}$ and so scales up to approximately $3 \mathrm{TeV}$ can be probed. In such models the $a_{\mu}$ measurement coupled with a $h \rightarrow \mu \mu$ branching ratio measurement could thus help elucidate the mechanism generating fermion masses.

In the models with a small value of $\mathrm{C}^{\mathrm{BSM}}(\sim O(\alpha / 4 \pi))$ a large contribution to $a_{\mu}$ is generally disfavoured and one would expect a measurement within 1-2 $\sigma$ of the SM. Conversely if a large value of $a_{\mu}$ is measured then a large class of such models would be ruled out. These include models with extra weakly-interacting gauge bosons $\left(\mathrm{Z}^{\prime}, \mathrm{W}^{\prime}\right)$, certain extra dimension models and variants of Little Higgs Models. For example the contributions to $a_{\mu}$ in a model with $\delta=1$ (or 2) universal extra dimensions [16] or a Littlest Higgs model with T-parity [17] are both less than $10 \times 10^{-11}$ i.e. below the expected precision of the FNAL measurement and would be ruled out if a large $a_{\mu}$ were measured. The signature of these models at the LHC can be very similar to SUSY models but SUSY models in general predict larger $a_{\mu}$. This is one of several examples where the $a_{\mu}$ measurement in concert with LHC observations can help resolve model degeneracy.

In the models with intermediate values of $\mathrm{C}^{\mathrm{BSM}}$ i.e. $O(\alpha / 4 \pi)<C^{\mathrm{BSM}}<O(1)$ then the mass scales of the BSM physics phenomena are generally around the weak scale. The most well-known example of such a model is SUSY where $a_{\mu}$ receives an enhancement proportional to $\tan \beta$ (the ratio of the vacuum expectation values of the two Higgs doublets). In SUSY models the predictions for $a_{\mu}$, the muon electric dipole moment and the rate of $\mu \rightarrow e$ conversion are determined by the 
slepton mixing parameters. These are poorly constrained by LHC data which are predominantly sensitive to SUSY squark and gluino parameters. Indeed present limits on the smuon mass are presently below $250 \mathrm{GeV}$. In the simplest SUSY models the SUSY contribution to $a_{\mu}$ is given by:

$$
a_{\mu}^{\mathrm{SUSY}}=\operatorname{sgn}(\mu) \times 130 \times 10^{-11} \times \tan \beta\left(\frac{100 \mathrm{GeV}}{\Lambda}\right)^{2}
$$

and so can provide sizeable contributions and indeed offers the potential for a much more precise determination of $\tan \beta$ than is possible from LHC data alone and also a determination of the sign of the SUSY $\mu$-parameter. With $100 \mathrm{fb}^{-1}$ of data the LHC can determine $\tan \beta$ with a precision of approximately $50 \%$, but when combined with a $0.14 \mathrm{ppm}$ measurement of $a_{\mu}$ it can be determined to $10 \%$.

The fact that no evidence for SUSY has been established at the LHC has resulted in lower limits for $\Lambda$ in the simplest SUSY models ranging from $300 \mathrm{GeV}$ to $1.5 \mathrm{TeV}$. The SUSY model builders trying to find SUSY parameters that are still allowed by the latest LHC and dark matter constraints find some tension with these and those favoured by the BNL $a_{\mu}$ measurement which favours somewhat lower SUSY masses in the range 200-700 GeV. However, given the large number of free parameters in SUSY models, there still remains significant regions of SUSY phase space that can accommodate a large value of $a_{\mu}$ and the LHC and dark matter constraints. This is illustrated in Fig. 2 where the ATLAS [18] (and LEP) limits on the smuon and neutralino mass for a certain choice of mSUGRA parameters are compared to the masses (for different values of $\tan \beta$ ) required to produce the $a_{\mu}$ value measured by E821 [19].

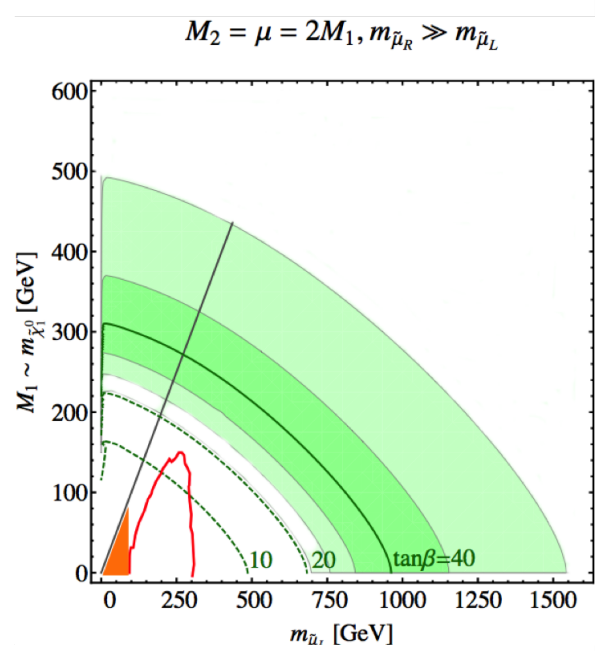

Figure 2: The ATLAS (red) and LEP (orange shaded) limits on the smuon and neutralino mass for a certain choice of mSUGRA parameters compared to the masses (for different values of $\tan \beta$ required to produce the $a_{\mu}$ value measured by E821. The darkest green shaded region represents the values required to give the E821 value $\pm 1 \sigma$ for a $\tan \beta$ value of 40 .

Recently particular attention has been paid to SUSY models where the squark and slepton mass scales are different. A priori there is no reason to expect that the coloured and non-coloured 
SUSY sectors should behave the same and in [20] it is stated: "Looking to (SUSY) models with a different connection between the coloured and uncoloured sector, not only seems timely now, but mandatory". Measurements, such as $a_{\mu}$, in the non-coloured sector which remain difficult at the LHC will clearly be vital in establishing the integrity of such models. Indeed many alternate SUSY models beyond the simple cMSSM are now being pursued such as natural-SUSY [21], gaugemediated SUSY breaking [22] and compressed SUSY [23] which can accomodate a large $a_{\mu}$ and the current LHC and dark matter constraints.

There are also many non-SUSY variants that predict sizeable contributions to $a_{\mu}$. The most well-know are large extra dimension models, variants of Randall-Sundrum models $[24,25]$ and unparticle models [26].

Recently a number of non-minimal models for the dark-matter sector have been explored where in analogy with ordinary matter coupling to the long-range EM force mediated by photons, a new long-range force that couples to dark-matter, mediated by "dark-photons" has been postulated. These dark-photons can be very light and, in some sense, be viewed as extremely light $Z^{\prime}$ bosons. While the dark-photons have no direct coupling to dark-matter, they can mix with the photon, thus inducing measurable effects in $a_{\mu}$ and also $a_{e}$. These models (for sub-GeV) particles are very difficult to study at the LHC but $a_{\mu}$ can probe masses in the 20-200 MeV mass region.

An improved measurement of $a_{\mu}$ will be vital in determining the validity of any of these models.

\section{The new g-2 experiment at FNAL (E989)}

The new experiment at FNAL will reduce the experimental uncertainty by over a factor of 4 with respect to the BNL measurement. This will be achieved in three ways:

- Utilising a well-established technique and apparatus (the original BNL storage ring).

- Increasing the number of stored muons by a factor of 21 to reduce the statistical uncertainty by more than a factor of 4 .

- Reducing the systematic uncertainty by a factor of 3 .

The BNL measurement was dominated by statistical uncertainties whereas the new FNAL measurement is expected to achieve parity between the statistical and systematic uncertainties.

The experimental measurement is in principle straightforward: positively-charged, polarised muons of a well-defined momentum $(3.094 \mathrm{GeV})$ are injected into a storage ring and the rate of precession of the muon's spin is determined by measuring the number of decay positrons (above an energy threshold) as a function of time coupled with a very precise measurement of the storage ring's $1.45 \mathrm{~T}$ magnetic field. The measurement exploits the parity-violation inherent in the weak interaction which means the direction of the muon spin and the electron decay direction are correlated in the muon's rest frame: specifically the highest energy $e^{+}$are emitted parallel to the $\mu^{+}$ spin in the muon rest frame. Muons in the storage ring are confined radially by the fixed $1.45 \mathrm{~T}$ dipole magnetic field and must be constrained vertically by an additional quadrupole electric field. This electric field would ordinarily also contribute to the rate of the muon's spin precession since 
the electric field is seen as a moving magnetic field in the muon's rest frame except at certain values of the muon momentum, $p$, when $p=\frac{m}{\sqrt{a_{\mu}}}$ which fortunately happens to be at a momentum, the so-called magic-momentum of $3.094 \mathrm{GeV}$, that is readily achievable in a particle accelerator. At this magic-momentum, the rate at which the muon precesses relative to the momentum, $\omega_{a}$, is simply given by: $-\frac{Q e}{m} a_{\mu} B$. Small (sub-ppm) corrections are applied to $\omega_{a}$ to account for the fact that not all muons in the storage ring are at the magic-momentum and not all muons have a trajectory exactly perpendicular to the magnetic field. $\omega_{a}$ is determined from a five-parameter fit to the function: $N\left(t, E_{t h}\right)=N_{0}\left(E_{t h}\right) e^{-t / \gamma \tau_{\mu}}\left[1+A\left(E_{t h}\right) \cos \left(\omega_{a} t+\phi\left(E_{t h}\right)\right)\right]$ which describes the number of decay positrons above an energy threshold, $E_{t h}$, detected as a function of time in terms of $\omega_{a}$, the muon lifetime $\tau_{\mu}$ and two parameters: $A$ and $\phi$ which are known functions of $E_{t h}$ and the polarization and momentum of the $\mu^{+}$beam. The optimum precision on $\omega_{a}$ is realised for threshold energies of approximately $1.8 \mathrm{GeV}$. Data is typically collected for approximately 10 muon lifetimes $\left(\gamma \tau_{\mu}=64 \mu \mathrm{s}\right.$ ) per muon spill. Fig. 3 shows $N(t)$ (modulo $100 \mu \mathrm{s}$ ) for 3.6 billion electrons with a threshold energy of $1.8 \mathrm{GeV}$ from the final E821 $\mu^{-}$dataset along with the five-parameter fit. The g- 2 precession period is $4.37 \mu$ s i.e. one precession is completed approximately every 30 revolutions of the storage ring.

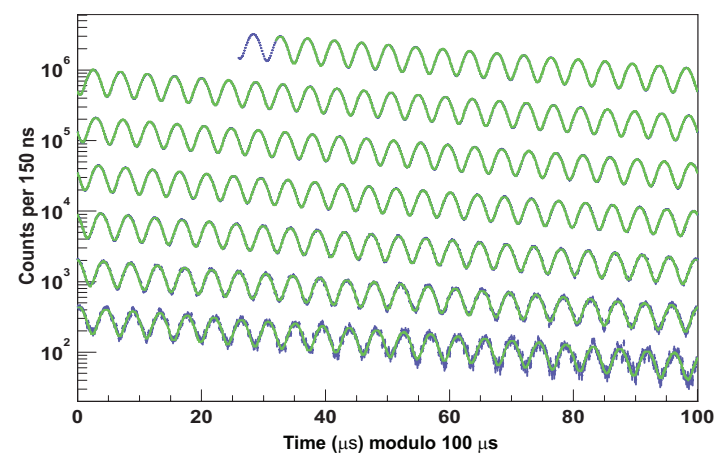

Figure 3: The number of decay electrons detected as a function of time (modulo $100 \mu \mathrm{s}$ ) from the E821 2001 dataset (blue) with a 5-parameter least-squares fit superimposed (green).

\section{Key improvements in the FNAL g-2 experiment}

The FNAL g-2 experiment will be improved in four keys ways over the BNL E821 experiment that will result in reductions in the statistical and systematic uncertainties by factors of 4 and 3 respectively. These improvements are:

- accelerator improvements to produce more muons per proton at a lower instantaneous rate with a far reduced pion contamination,

- a new inflector and kicker magnet resulting in a larger acceptance, a more stable beam and better damping of the coherent betatron oscillations, 
- improved uniformity and calibration of the B-field,

- new detectors with improved stability, resolution and better handling of pileup.

These improvements are described in more detail in the following sections.

\subsection{Accelerator improvements}

Compared to BNL the FNAL accelerator complex will produce more muons at a lower instantaneous rate with a far reduced pion contamination. This is being achieved by constructing new muon beamlines that will serve both the $\mathrm{g}-2$ and Mu2e experiments in a dedicated muon campus. Modifications to the proton accelerators are already largely complete as part of the improvements necessary for the NOvA and MicroBoone experiments. These include a new RFQ injector into the LINAC, an increase in the FNAL Booster repetition rate (ultimately to $15 \mathrm{~Hz}$ ) and a new connection between the Booster and The Recyler. The g-2 experiment will run concurrently with the neutrino experiments and will use 4 from the 20 Booster proton batches injected from the LINAC (per Main Injector cycle). Each of these four proton batches (separated by $132 \mathrm{~ms}$ ) is further split into 4 micro-bunches (of $\sim 100 \mathrm{~ns}$ duration and separated by $12 \mathrm{~ms}$ ) such that on average the rate of muon"spills" injected into the storage ring is $12 \mathrm{~Hz}$ with each spill containing $\sim 10^{5}$ muons. With an assumed uptime and data-taking efficiency of 75\%, a data-taking period of 14 months (plus 2 months for commissioning and 2 for systematic studies) is required to achieve the required statistical precision $(0.1 \mathrm{ppm})$.

The muon beam will be produced in the complex that was used by the Tevatron to produce antiprotons and new beamlines will be constructed to the $\mathrm{g}-2$ and Mu2e experiments. Protons will be accelerated to a kinetic energy of $8 \mathrm{GeV}$ in the Booster and re-bunched into the four smaller (micro-)bunches in the Recycler with $\sim 10^{12}$ protons per bunch and transported through three beamlines (P1, P2 and M1) to the old antiprototon production target. A secondary beam from the target will be focussed using a lithium lens and positively-charged particles with a momentum of $3.11 \mathrm{GeV}( \pm \sim 10 \%)$ will be selected using a bending magnet. The secondary beam leaving the target station will travel through two beamlines (M2 and M3) which are designed to maximise the capture of muons with momentum $3.094 \mathrm{GeV}$ from pion decays. The beam will then be injected into the Delivery Ring. After several revolutions around the Delivery Ring essentially all of the pions will have decayed into muons and the muons will also have separated in time from the heavier protons. A kicker will then be used to abort the protons, and the muon beam will be extracted into a new beamline (M5) that leads to the g-2 storage ring which will be housed in a dedicated building (MC-1). Several of the beamlines and the associated cryogenics will also ultimately be used to transport $8 \mathrm{GeV}$ protons to the Mu2e experiment. After the proton target and before the (g-2) ring the particles traverse almost $2 \mathrm{~km}$ of beamline which ensures that the beam entering the g- 2 storage ring is essentially pion-free which is a significant improvement over the BNL experiment. The beamlines serving the muon campus are shown in Fig. 4. Construction of the experimental hall for the g-2 storage ring has begun and it will be ready for occupancy in March 2014. This building will provide a much more stable environment for the experiment in terms of alignment and temperature compared to the BNL experiment. 


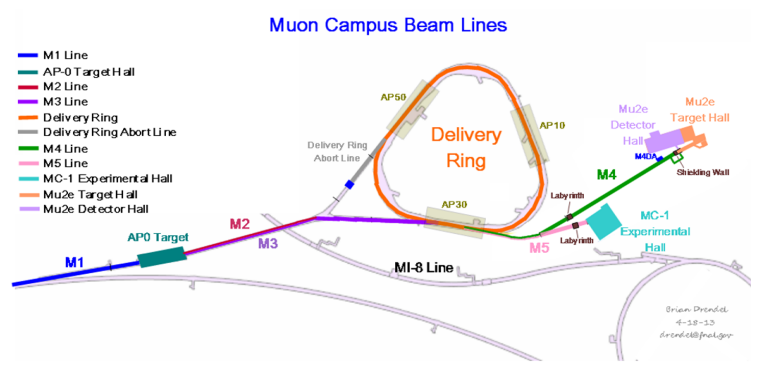

Figure 4: The FNAL Muon Campus beamlines and experimental halls. MC-1 will house the g-2 storage ring.

\subsection{Injection/beam-storage improvements}

The muon beam is injected into the storage ring using a novel superconducting "inflector" magnet that cancels the main $1.45 \mathrm{~T}$ field from the storage-ring and the beams enters the ring displaced by $77 \mathrm{~mm}$ with respect to the desired central orbit. They are placed into the central orbit by a fast-kicker pulsed-magnet that moves the beam through $14 \mathrm{mrad}$. The requirements on the inflector magnet are very restrictive: it should null the storage ring field such that the muons are not deflected by the main $1.45 \mathrm{~T}$ field; it should be a static device to prevent time-varying magnetic fields correlated with injection; it cannot "leak" magnetic flux into the precision shimmed storagering field and as such cannot contain any ferromagnetic materials.

The E821 experiment used a truncated double $\cos \theta$ magnet [27] with equal and opposite currents in two concentric magnets and a superconducting shield that reduced the "leaked" magnetic flux from the inflector to a level that induced only a $0.2 \mathrm{ppm}$ systematic on the determination of the B-field. This is the baseline design for the FNAL inflector but three key improvements will also be implemented:

- A reduction in the flux leakage e.g. through careful design of the current-carrying leads, to below $1 \mathrm{G}$ such that the B-field systematic can be reduced from $0.2 \mathrm{ppm}$ to $0.07 \mathrm{ppm}$.

- An opening in the ends of the magnet and a larger beam aperture to improve the beam stability and increase the number of stored muons by a factor of 3.8 .

- Improved beam matching in the injection system to reduce beam losses and coherent betatron oscillations (CBO).

A prototype of the open-inflector developed for E821 is shown in Fig. 5.

A new fast-kicker magnet system will also be produced. In the BNL experiment the kicker pulse was not a flat-top and had a duration longer than the cyclotron period of $149 \mathrm{~ns}$ such that a muon pulse received more than a single "kick". A new kicker will address these limitations in the BNL kicker and will provide an integrated vertical field of 1.1-1.4 kG m from three independent $1.7 \mathrm{~m}$ long magnets, each with a dedicated pulse forming network. The kicker hardware cannot contain magnetic elements such as ferrites since they will perturb the storage-ring's field and any eddy currents must be negligible (or well-known) within $20 \mu$ s after injection when the positron 

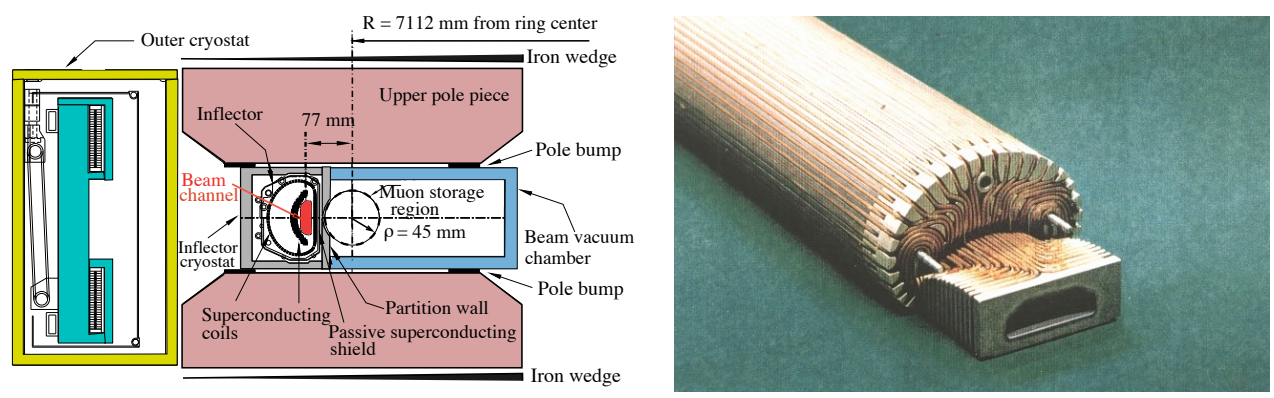

Figure 5: (LEFT): A cross sectional view of the inflector and storage-ring dipole. (RIGHT): A prototype of the open-inflector developed for E821.

decays begin to be recorded. The new kicker will be based on a Blumlein triaxial transmission line as opposed to the E821 LCR PFN design and a prototype is presently under test at Cornell.

\subsection{Improvements in the B-field measurement}

The determination of $a_{\mu}$ requires both the measurement of $\omega_{a}$ from the modulation in the arrival time of the decay positrons and the B-field. In particular one needs to determine the integrated B-field experienced by the muons and so this necessarily also involves measurements (and simulation) of the muon beam's profile and trajectory. The muon beam is confined to a cylindrical region of $9 \mathrm{~cm}$ in diameter and $44.7 \mathrm{~m}$ in length such that the field must be known over a volume of approximately $1.1 \mathrm{~m}^{3}$. To achieve the factor of three reduction in the systematic uncertainty the $1.45 \mathrm{~T}$ storage-ring magnetic field must be determined to an accuracy of $0.07 \mathrm{ppm}$. This will be achieved through: improved shimming of the magnet and temperature control, an increase in the frequency at which the field is mapped and the area which is mapped through improved probe positioning. Improvements in the measurement of the profile and trajectory will come from an improved simulation of the accelerator optics and through the introduction of two straw tracking detectors (see section 5.4).

The storage-ring's $1.45 \mathrm{~T}$ magnetic field is provided by three superconducting coils. A continuous "C-shape" yoke is formed from twelve $30^{\circ}$ segments of iron designed to minimise the impact of edge-effects on the field. This formation eliminates large gradients that would otherwise make a precise determination of the B-field very difficult. The coils and yoke will be those used in the E821 experiment.

The mapping of the field will be achieved using: an array of 378 NMR probes outside of the vacuum chamber that monitors the magnetic field during data collection; a mapping trolley of 17 NMR probes moved through the experimental volume 2-3 times per week (without beam) and plunging probes to make additional measurements. The homogeneity in the field achieved in E821 
is shown in Fig. 6 where it can be seen for the central region that the field is uniform to better than $0.5 \mathrm{ppm}$.

The absolute calibration (relative to the free-proton) is provided by a spherical water probe. This provides a measurement of the magnetic field in terms of the Larmor frequency of a free proton, $\omega_{p}$. This is combined with the $\omega_{a}$ measurement to determine $a_{\mu}$ via the relation: $a_{\mu}=\frac{\omega_{a} / \omega_{p}}{\lambda-\omega_{a} / \omega_{p}}$, where $\lambda$ is the muon-to-proton magnetic moment ratio determined from hyperfine splitting measurements in muonium. The FNAL absolute calibration water probe will be checked against the absolute calibration probe to be used for the J-PARC muonium hyperfine splitting (MuHFS) experiment $[28,29]$ that will measure $\lambda$ to a precision of $0.01 \mathrm{ppm}$.
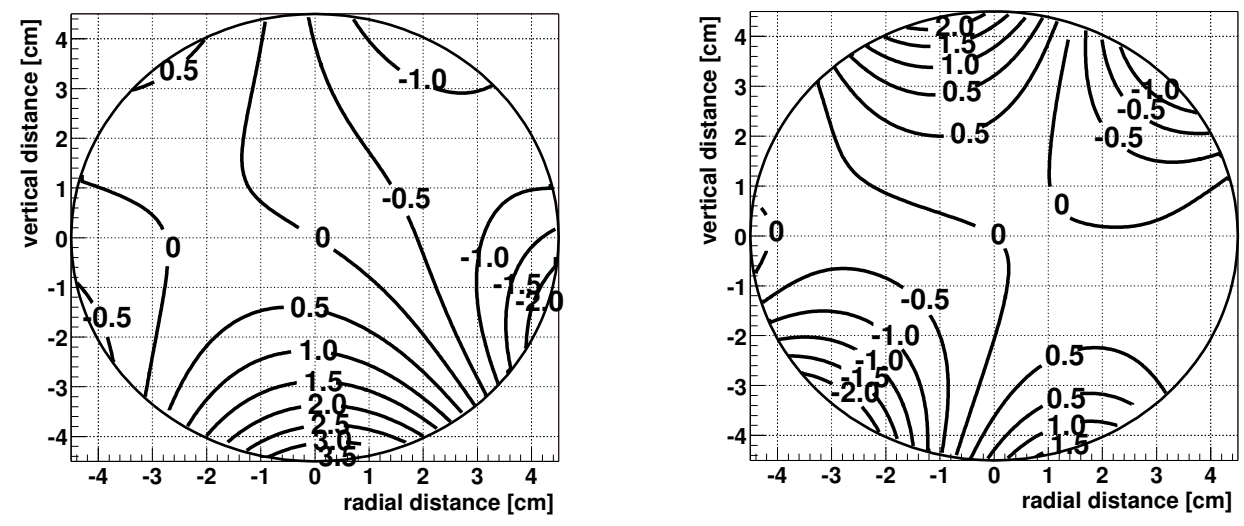

Figure 6: Homogeneity of the field in E821: at the calibration position (LEFT) and for the azimuthal average for one trolley run during the 2000 period (RIGHT). In both figures, the contour spacing is $0.5 \mathrm{ppm}$.

\subsection{Improvements in the detectors}

$\omega_{a}$ is determined from the modulation in the arrival time of the decay positrons above an energy threshold. This is subject to uncertainties arising from the detector calibration and from pileup whereby two low energy positrons overlap spatially and temporally in the calorimeter. The pileup affects the pulse fitting algorithm and may cause two low energy positrons to fake a high energy positron. Changes in the detector gain over the duration of the fill are particularly critical since they will further modulate the time distribution of the positrons.

Several improvements will be implemented to allow a reduction in the gain and pileup systematic uncertainties by a factor of 3 . These include:

- A new $\mathrm{PbF}_{2}$ crystal calorimeter with improved segmentation and resolution.

- Calorimeter readout using SiPMs which are always on i.e. due to the reduced pion contamination there is no need to turn off electronics during injection.

- Improved temperature (and hence gain) control.

- Better monitoring of the calorimeter gain using an UV laser. 
- The recording of all calorimeter samples (not just those above a threshold).

- A large acceptance straw tracker system that will provide high precision tracking with good momentum resolution.
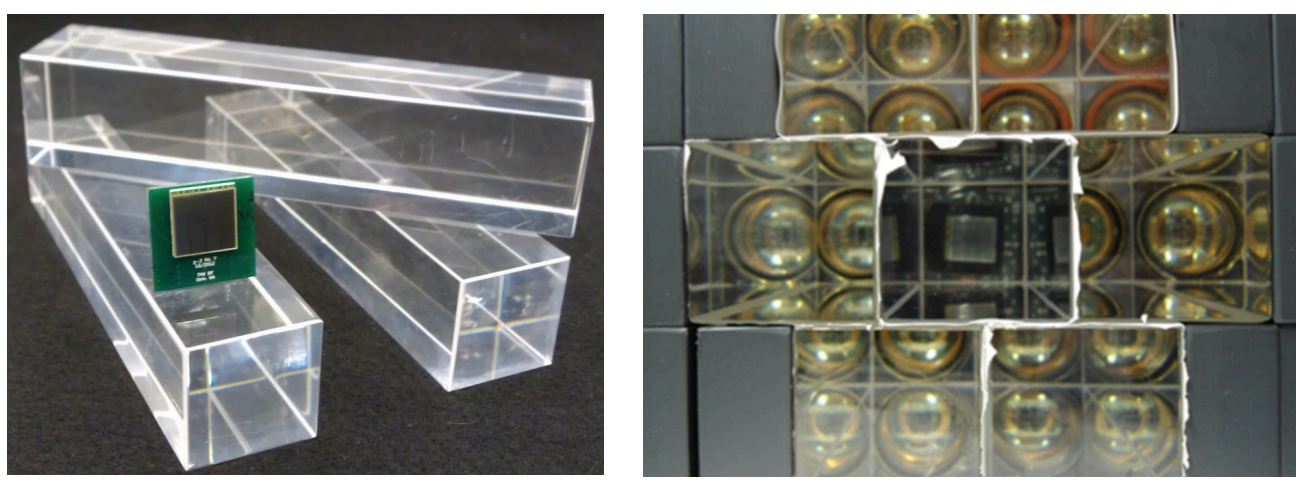

Figure 7: (LEFT): A close-up of prototype $\mathrm{PbF}_{2}$ crystals together with a 16-channel Hamamatsu SiPM. (RIGHT): The 7-crystal $\mathrm{PbF}_{2}$ array used in the FNAL test beam. The SiPM is visible on the center channel, while conventional PMTs were used on the remaining crystals.

$\mathrm{PbF}_{2}$ can achieve a resolution $(5 \%$ at $2 \mathrm{GeV})$ a factor of two better than the E821 calorimeter with a fast Cherenkov signal, a Moliere radius of $1.3 \mathrm{~cm}$ and critically it has a very low magnetic susceptibility. A prototype of the $\mathrm{PbF}_{2}$ calorimeter and $\mathrm{SiPM}$ readout have recently been tested in a testbeam at FNAL. The array used is shown in Fig. 7.

The in-vacuum straw tracker will be used to provide calibration via $E / p$ and to identify pileup events so that calorimeter pulse-fitting algorithms can be optimised and any biasses quantified. The tracker will also be used to determine the muon beam profile that is important for the determination of $\omega_{p}$. Each tracker will be composed of $\sim 10$ separate stations and will measure the momentum to better than $3.5 \%$ at $1 \mathrm{GeV}$, have a vertical angular resolution of better than $10 \mathrm{mrad}$ and determine the impact parameter of the tracks to better than $1 \mathrm{~cm}$. The straw design is based on one for Mu2e and prototype detectors (see Fig. 8) will be evaluated in a testbeam in February 2014.

The straw trackers in addition to reducing the systematics in the $a_{\mu}$ measurement will also be used to make a measurement of the muon's electric dipole moment (EDM) by measuring the average vertical angle of positron tracks versus time (modulo the precession frequency). In the SM the muon EDM is predicted to be $O\left(10^{-35}\right) \mathrm{e} \mathrm{cm}$ and simple mass scaling of the most recent limit on the electron EDM [30] would suggest the muon EDM is $<O\left(10^{-26}\right) \mathrm{e} \mathrm{cm}$. The addition of the straw trackers will increase the sensitivity to the EDM by a factor of 100 compared to E821 down to $10^{-21} \mathrm{e} \mathrm{cm}$. Any non-null observation would be evidence for a new CP-violating interaction. The measurement can probe models where the CP-violating phases are flavour dependent e.g. those with non-trivial mass scaling such as Randall-Sundrum extra dimensions [32] that allow for a muon 


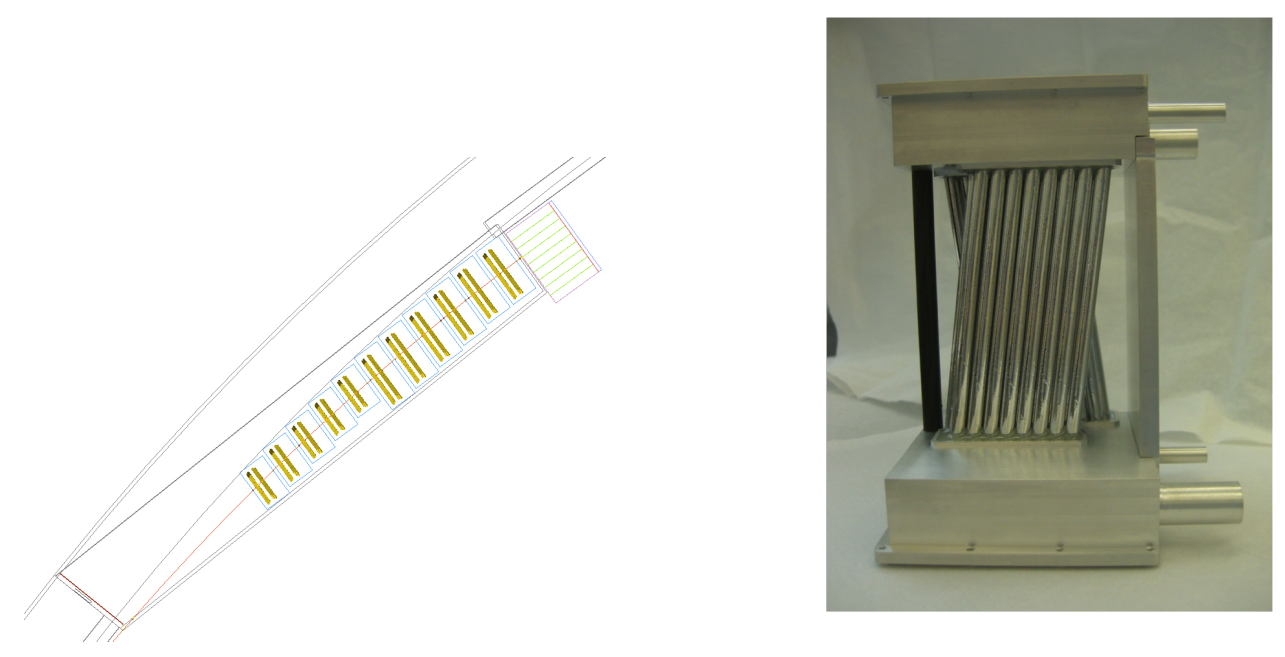

Figure 8: (LEFT): The placement of the straw tracking stations in the vacuum region in front of a calorimeter. (RIGHT): A prototype tracking station comprising a doublet of (U,V) straws.

EDM within 1 order of magnitude of the current limit [31] and thus within the sensitivity reach of the FNAL experiment.

\section{Current Status and Schedule}

The experiment has recently been granted CD-1 approval by the DOE and the final technical decisions will be made in the summer of 2014. The civil construction of the experimental hall will be completed in February 2014. The infrastructure from the E821 experiment that will be re-used for the FNAL experiment has already been shipped from BNL to FNAL. In particular the storage ring was successfully transported in the summer of 2013. The journey of 3,200 miles was undertaken by truck and barge and took 34 days. A photograph of the barge in transit and arriving at FNAL is show in Fig. 9.

The current schedule anticipates having the storage ring and yokes reassembled and in the experimental hall for a test with power and cryogenics at the start of 2015. Testbeams for the detectors will continue throughout 2014. In 2015 the muon beamlines will be commissioned and the storage ring shimmed. The vacuum chambers and detectors will be installed in 2016 and first data is expected before the end of 2016. A data-taking (plus calibration) period of 18 months is required to reach the statistical goal $(0.07 \mathrm{ppm})$. The accelerator, injection, field and detector improvements should also allow a systematic uncertainty of $0.07 \mathrm{ppm}$ to be achieved which will result in a measurement over 4 times more precise than the BNL measurement.

On the same timescale new data and analyses of low energy $e^{+} e^{-}$cross section data will be available allowing a reduction in the uncertainty in the SM prediction for $a_{\mu}$. By the end of decade there will thus be clarity on whether the $3.5 \sigma$ anomaly observed by the E821 experiment is indeed a harbinger of new physics or whether the SM passes one of its most precise tests. 

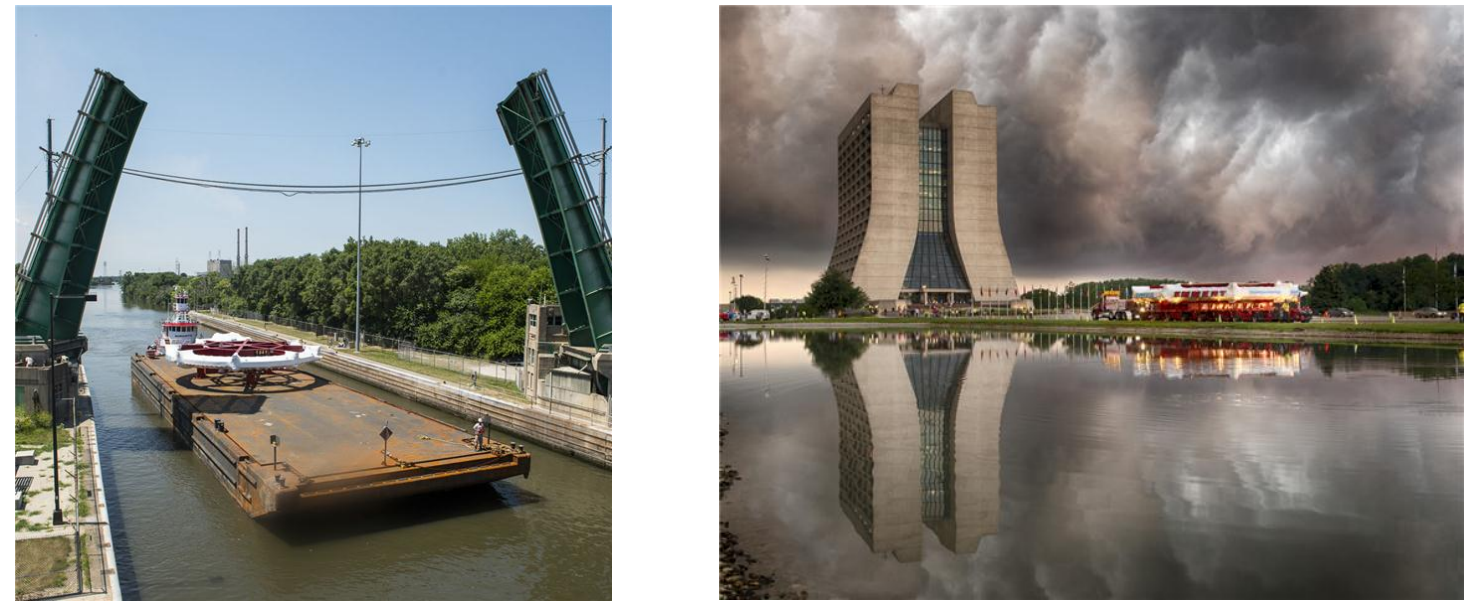

Figure 9: (LEFT): The storage ring on the Illinois river close to the end of its 3,200 mile journey from BNL to FNAL. (RIGHT): The storage ring shortly after its arrival at FNAL in July 2013.

\section{Acknowledgements}

I would like to thank the organisers of Photon-2013 for a supremely well organised, stimulating and enjoyable conference and the STFC for their financial support that enabled me to present the status of the FNAL g-2 experiment at this conference. I would also like to thank Frank Deppisch for insightful discussions and for providing Fig.2.

\section{References}

[1] E821 Collaboration, G. W. Bennett, et al., Phys. Rev. Lett. 92, 161802 (2004).

[2] M. Knecht, Status of SM calculation of lepton g-2, these proceedings, PoS (Photon 2013) 040.

[3] J. Schwinger, On Quantum-Electrodynamics and the Magnetic Moment of the Electron, Phys. Rev. 73, 416 (1948).

[4] T. Aoyama et al., Complete Tenth-Order QED Contribution to the Muon g-2, Phys. Rev. Lett. 109, 111808 (2012).

[5] A. Kurza, T. Liu, P. Marquard and M. Steinhauser Anomalous magnetic moment with heavy virtual leptons, Nucl. Phys. B 879, 1 (2014).

[6] C. Gnendiger, D. Stöckinger and H. Stöckinger-Kim, The electroweak contributions to $(g-2)_{\mu}$ after the Higgs-boson mass measurement, Phys. Rev. D 88, 053005 (2013).

[7] A. Passeri, Measurement of hadronic cross sections at KLOE with ISR and limits on their impact to the muon anomaly and U-boson search, these proceedings, PoS (Photon 2013) 043.

[8] B. Malaescu, Measurement of hadronic cross sections at BaBar with ISR and implications for the muon $(g$-2), these proceedings, PoS (Phot on 2013) 044 .

[9] S. Eidelman, Status and prospects of e+e-hadronic cross sections at low energy, these proceedings, PoS (Photon 2013) 045.

[10] I. P. Longhi, Measurements of the $\pi^{0} \rightarrow$ gg decay width and the $\pi^{0}$ transition form factor at KLOE-2 and its impact on HLbL, these proceedings, PoS (Photon 2013) 046. 
[11] Z. Zhang, Review of recent Leading-Order Hadronic Vacuum Polarization calculation, these proceedings, PoS (Photon 2013) 047.

[12] A. Vainshtein, Hadronic Light-by-Light Scattering in the Muon Anomalous Magnetic, these proceedings, POS (Photon 2013) 050.

[13] M. Baak et al., The Electroweak Fit of the Standard Model after the Discovery of a New Boson at the LHC, Eur. Phys. J. C 72, 2205 (2012).

[14] M. Passera, W. J. Marciano and A. Sirlin, The muon g-2 discrepancy: errors or new physics ?, AIP Conf. Proc. 1078, 378 (2009).

[15] A. Czarnecki and W. J. Marciano, Muon anomalous magnetic moment: A harbinger for "new physics”, Phys. Rev. D 64, 013014 (2001).

[16] T. Appelquist and B. A. Dobrescu, Universal extra dimensions and the muon magnetic moment, Phys. Lett. B 516, 85 (2001).

[17] M. Blanke et al., Charged Lepton Flavour Violation and $(g-2)_{\mu}$ in the Littlest Higgs Model with T-Parity: A Clear Distinction from Supersymmetry, JHEP 0705, 013 (2007).

[18] ATLAS Collaboration, Searches for direct slepton-pair and chargino-pair production in final states with two opposite-sign leptons, missing transverse momentum and no jets in $20 \mathrm{fb}^{-1}$ of pp collisions at $\sqrt{s}=8 \mathrm{TeV}$, ATLAS-CONF-2013-049 (2013).

[19] F. Deppisch, private communication.

[20] O. Buchmueller et al., The CMSSM and NUHM1 in Light of 7 TeV LHC, Bs to $\mu^{+} \mu^{-}$and XENON100 Data, Eur. Phys. J. C 72, 2243 (2012).

[21] V. Barger, M. Ishida and W-Y. Keung, Flavor-Tuned 125 GeV SUSY Higgs Boson at the LHC: MSSM and Natural SUSY Tests Phys. Rev. D 87, 015003 (2013).

[22] M. Endo, K. Hamaguchi, S. Iwamoto and N. Yokozaki, Higgs mass, muon g-2, and LHC prospects in gauge mediation models with vectorlike matters, Phys. Rev. D 85, 095012 (2012).

[23] T. LeCompte and S. Martin, Compressed supersymmetry after $1 \mathrm{fb}^{-1}$ at the Large Hadron Collider, Phys. Rev. D 85, 035023 (2012).

[24] C. S. Kim, J. D. Kim and J. Song, Muon anomalous magnetic moment (gấL Š2) Îij and the RandallâĂŞSundrum model, Phys. Lett. B 511, 251 (2001).

[25] M. Beneke, P. Dey and J. Rohrwild, The muon anomalous magnetic moment in the Randall-Sundrum model, JHEP 08, 010 (2013).

[26] A. Hektor, Y. Kajiyama and K. Kannike, Muon Anomalous Magnetic Moment and Lepton Flavor Violating Tau Decay in Unparticle Physics, Phys. Rev. D 78, 053008 (2008).

[27] A. Yamamoto et al., The superconducting inflector for the BNL g-2 experiment, Nucl. Instr. Methods A 497, 23 (2002).

[28] K. Sasaki et al., Development status of superconducting solenoid for the MuHFS experiment at the J-PARC, J. Phys. Conf. Ser. 408, 012074 (2013).

[29] A. Toyoda et al., J-PARC MUSE H-line optimization for the g-2 and MuHFS experiments, J. Phys. Conf. Ser. 408, 012073 (2013).

[30] The ACME Collaboration, J. Baron et al., Order of Magnitude Smaller Limit on the Electric Dipole Moment of the Electron, Science 1248213, (2013). 
[31] G. W. Bennett et al., An Improved Limit on the Muon Electric Dipole Moment, Phys. Rev. D 80, 052008 (2009).

[32] E. O. Iltan, The effects of lepton KK modes on the lepton electric dipole moments in the Randall Sundrum scenario, Eur. Phys. J. C 54, 583 (2008). 